\title{
Traffic-Related Air Pollutants Induce the Release of Allergen-Containing Cytoplasmic Granules from Grass Pollen
}

\author{
A.C. Motta ${ }^{a}$, M. Marliere ${ }^{c}$, G. Peltre ${ }^{\text {d }}$, P.A. Sterenberg ${ }^{\text {b }}$, G. Lacroix ${ }^{c}$ \\ aLaboratory of Allergology and Pulmonary Diseases, Department of Pathology and \\ Laboratory Medicine, Groningen University Medical Center, Groningen University, \\ Groningen, and \\ ${ }^{b}$ National Institute of Public Health and the Environment, Bilthoven, The \\ Netherlands; \\ ${ }^{c}$ Institut National de I'Environnement Industriel et des Risques, Verneuil-en- \\ Halatte, and \\ ${ }^{\mathrm{d}}$ ESPCI Laboratoire Environnement et Chimie Analytique, Allergie et \\ Environnement, Paris, France \\ Correspondence to: Dr. Alexandre C. Motta \\ Laboratory of Allergology and Pulmonary Diseases, Department of Pathology and \\ Laboratory Medicine, Groningen University Medical Center, POB 30001 \\ NL-9700 RB Groningen (The Netherlands) \\ Tel. +3150 361 9530, Fax +31 50363 2510, E-Mail a.motta@path.umcg.nl
}

DOI: $10.1159 / 000091600$

\begin{abstract}
Background/Aim: Pollen cytoplasmic granules (PCG) are loaded with allergens. They are released from grass pollen grains following contact with water and can form a respirable allergenic aerosol. On the other hand, the traffic-related air pollutants $\mathrm{NO}_{2}$ and $\mathrm{O}_{3}$ are known to be involved in the current increase in the prevalence of allergic diseases via their adjuvant effects. Our objective was to determine the effects of air pollutants on the release of PCG from Phleum pratense (timothy grass) pollen. Methods:P. pratense pollen was exposed to several concentrations of $\mathrm{NO}_{2}$ and $\mathrm{O}_{3}$. The induced morphological damages were observed by environmental scanning electron microscopy, and the amount of PCG released from the pollen upon contact with water was measured. Results: The percentages of damaged grain were $6.4 \%$ in air-treated controls, $15 \%$ after treatment with the highest $\mathrm{NO}_{2}$ dose $(50 \mathrm{ppm})$ and $13.5 \%$ after exposure to 0.5 ppm $\mathrm{O}_{3}$. In treated samples, a fraction of the grains spontaneously released their PCG. Upon subsequent contact with water, the remaining intact grains released more PCG than pollen exposed to air only. Conclusions: Traffic-related pollutants can trigger the release of allergen-containing granules from grass pollen, and increase the bioavailability of airborne pollen allergens. This is a new mechanism by which air pollution concurs with the current increase in the prevalence of allergic diseases.
\end{abstract}

\section{Introduction}

Raising atmospheric pollution levels is one of the possible explanations for the current increase in the prevalence of respiratory allergic diseases. The trafficrelated gaseous pollutants $\mathrm{O}_{3}$ and $\mathrm{NO}_{2}$ are known for their toxicity on respiratory tracts, as well as for their adjuvant effect on respiratory hypersensitivity and on asthma $[1,2,3,4,5]$. Moreover, these pollutants can also interact with airborne pollen grains and modify their allergen content and release [6, 7]. Phleum pratense pollen is one of the most common sources of outdoor allergens. 
Although it is too big $(35 \mu \mathrm{m})$ to penetrate the airways [8], it can release cytoplasmic granules (CG) following contact with water [9]. Due to their small size $(3 \mu \mathrm{m})$, these granules can form a respirable aerosol capable of reaching the deep airways. Previously, we have shown that Phleum pratense pollen CG (PCG) contain allergens and can trigger a secondary allergic response in pollensensitized rats [10]. In addition, purified PCG were able to prime for specific IgE and T-cell responses in a rat model of allergy [11]. A dramatic example of the allergenic potential of PCG is the so-called thunderstorm-associated asthma. During these episodes, PCG are released from the pollen grains by contact with rainwater. Intact and emptied pollen grains are washed down by the rain but PCG, due to their small size, are carried by the wind, and a PCG-enriched cloud is formed, which moves along the front of the thunderstorm $[12,13,14]$. Pollenallergic patients who encounter this cloud would be more susceptible to undergo an asthma attack. The aim of our study was to determine if the traffic-related pollutants $\mathrm{O}_{3}$ and $\mathrm{NO}_{2}$, by damaging the pollen grains, could also significantly increase the amount of PCG released and thus have an impact on the quantity of allergens in the airborne submicronic fraction. We exposed pollen grains to several concentrations of gaseous pollutants in a fluidization chamber. Pollen grains were then observed with an environmental scanning electron microscope (ESEM) to determine the proportion of damaged grains, and we measured the changes in PCG release following contact with water.

\section{Methods}

Pollen Grains. Pollen grains from P. pratense (timothy grass) were purchased from Allergon, Angelholm, Sweden.

Pollen Exposure to Pollutants. The system was composed of a glass cylinder (height: $250 \mathrm{~mm}$, and diameter: $20 \mathrm{~mm}$ ) with a porous glass plate at the bottom. The pollen (about $2 \mathrm{~g}$ ) was placed in the cylinder and formed a layer of 25-30 $\mathrm{mm}$, lying on the porous glass plate. Mixtures of air and pollutants $\left(\mathrm{NO}_{2}: 0,0.5\right.$, $2,10,20$ and $50 \mathrm{ppm}$, and $\mathrm{O}_{3}: 0,0.1,0.25,0.5,1.3$ and $5 \mathrm{ppm}$ ) were injected from the bottom at a constant rate of $1.2 \mathrm{l} / \mathrm{min}$ for $4 \mathrm{~h}$. The pollen was then suspended in a rising current of air, producing a fluidized bed. Fluidization, ensuring homogenous exposure of the pollen, was obtained by a slight contact of the cylinder with a vortex.

Environmental Scanning Electron Microscopy. Pollen grains exposed to air or to pollutants were directly observed without treatment, in low vacuum mode $(0.7$ Torr), using an ESEM (Quanta 400, Philips). The proportion of damaged grains was determined by visual counting. A grain was considered damaged if visible cracks of the exine were present or if it was broken ( $n=1,000$ grains).

Quantification of Pollen Cytoplasmic Granule Release. PCG were isolated from pollen grains according to a protocol described previously [11]. Samples of nontreated, air-treated or pollutant-treated pollen $(10 \mathrm{mg})$ were suspended in $500 \mu \mathrm{l}$ distilled water to allow the release of PCG from remaining intact pollens. The suspension was then filtered by centrifugation through a 5- $\mu \mathrm{m}$ mesh filter at $10,000 \mathrm{~g}$ for $5 \mathrm{~min}$ (Ultrafree-MC; Millipore, Bedford, Mass., USA). PCG pellet was resuspended in $500 \mu \mathrm{l}$ distilled water and particles were counted with a particle count and size analyzer (Z2; Coulter, Hialeah, Fla., USA).

Statistical Analysis. The variable 'damaged grains' was considered to follow a binomial distribution. Results are expressed as the percentage of damaged grains \pm confidence interval of probability, estimated under the binomial distribution assumption. The amount of PCG released is considered as normally distributed. 
Results are expressed as means \pm SEM. Comparison between air-treated and pollutant-treated pollen was carried out using analysis of variance followed by the Dunett test. The differences were considered statistically significant for $p<0.01$.

\section{Results}

\section{Modifications of the Pollen Morphology following Exposure to Pollutants}

Exposure to air or pollutants damaged the pollen grains. ESEM revealed either fissures in the exine or complete breaking of the grains (fig. 1). Non-treated pollen already contained $3.4 \%$ damaged grains. Treatment with air only increased this proportion to $6.4 \%$. Exposure to pollutants induced damages of the grains in a dose-dependent way for $\mathrm{NO}_{2}$, (from $6.4 \%$ at $2 \mathrm{ppm}$ to $15 \%$ at $50 \mathrm{ppm}$ ) and non-dose-dependent manner for $\mathrm{O}_{3}$, reaching a plateau from $0.5 \mathrm{ppm}(13.5 \%)$. A dramatic increase up to $19.5 \%$ was observed for the highest $\mathrm{O}_{3}$ concentration ( 5 ppm).
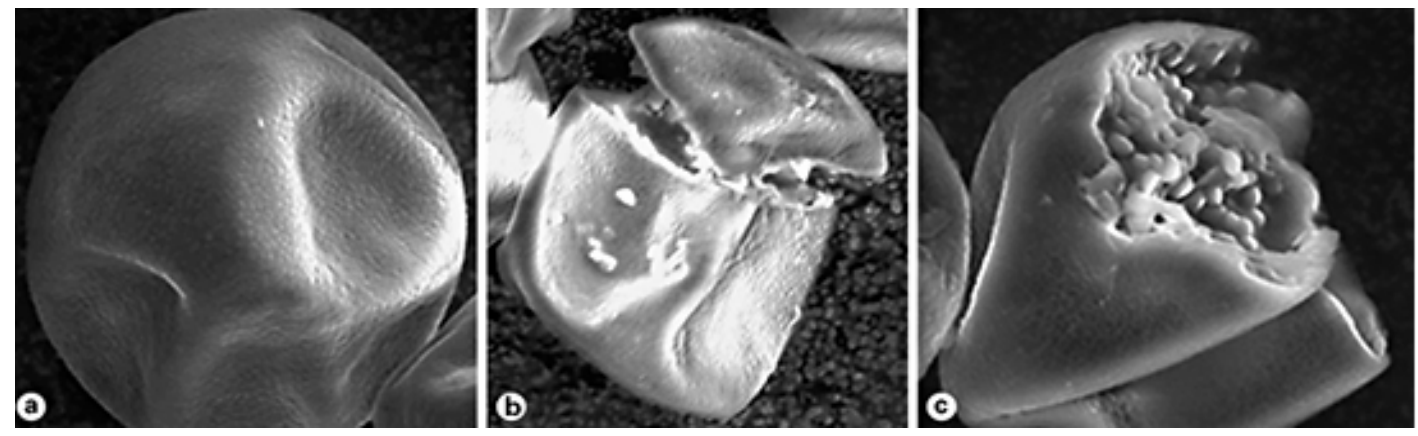

Fig. 1. Examples of damaged pollen grains. Treatment of pollen samples to air or pollutants can induce structural damage of the grains. a Intact pollen. $\times 7,540$. $\mathbf{b}$ Pollen damaged following treatment to $50 \mathrm{ppm} \mathrm{NO} \mathrm{NO}_{2} \times 3,000$. c Pollen damaged following treatment to $0.7 \mathrm{ppm} \mathrm{O}_{3}$. PCG can be seen inside the broken grain. $\times 6,000$.

\section{Modifications of PCG Release following Exposure to Pollutants}

PCG are naturally released from the pollen grain when the cytoplasm is expelled from the grain through the pore, following contact with water (fig. 2a, b). However, we observed that the release could also occur through cracks of the exine when the grain was damaged (fig. 2c). To look for pollutant-induced modifications in the PCG release, we isolated the granules liberated from $10 \mathrm{mg}$ of treated or non-treated samples upon contact with water and counted them with a particle counter. In all samples, size distribution of PCG presented in a curve ranged from 2.5 and $4 \mu \mathrm{m}$ in diameter, with a peak at $3 \mu \mathrm{m}$. Treatment with air only induced a dramatic decrease in the amount of PCG released when compared to non-treated pollen. This decrease was correlated with an increase in broken grains in the sample (fig. 3), indicating that the grains that had been broken during the exposure released their PCG, which were then carried out of the exposure system with the airflow. Indeed, PCG were present on a $0.45-\mu \mathrm{m}$ mesh filter placed at the exit of the exposure system in a control experiment. Only the remaining intact grains could release their PCG upon a subsequent contact with water. Among these intact grains, only a small proportion was able to release PCG [15]. In pollutant-treated samples, the proportion of damaged pollen grains was higher than in air-treated samples, and thus less intact grains remained. Interestingly, the amount of PCG released after contact with water 
increased in pollutant-treated samples. Consequently, among the remaining intact grains, the proportion of grains susceptible to expel their cytoplasm was increased. $\mathrm{NO}_{2}$ treatment induced a dose-dependent increase in the PCG amounts released, being statistically significant from 2 to $50 \mathrm{ppm}$. Following $\mathrm{O}_{3}$ treatment, the increases in the PCG amounts were statistically significant from the lowest $(0.1 \mathrm{ppm})$ to the highest concentration $(5 \mathrm{ppm})$. This increase remained stable for the three first concentrations $(0.1,0.25$ and $0.5 \mathrm{ppm})$ and rose dramatically at $1.3 \mathrm{ppm}$. For both pollutants, the amount of PCG released showed a sharp decrease at the highest concentration ( $50 \mathrm{ppm} \mathrm{NO} \mathrm{N}_{2}$ and $5 \mathrm{ppm} \mathrm{O}_{3}$ ). This is probably due to the fact that, in these samples, too much PCG were lost during the treatment via spontaneous release as indicated by the concomitant peaks of damaged grains.
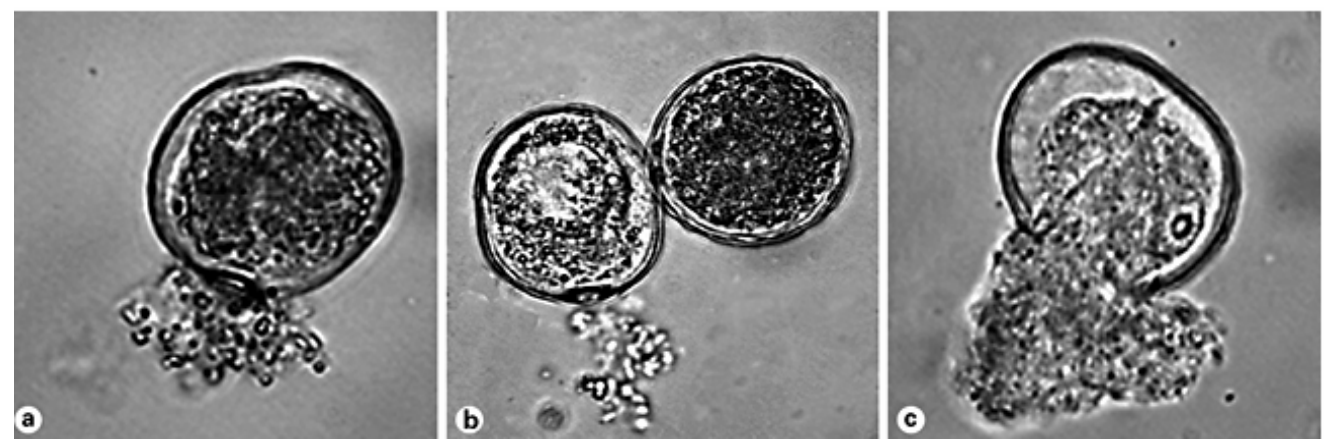

Fig. 2. Release of PCG following contact of pollen grains with water. PCG are expelled from the grain via the pore $(\mathbf{a}, \mathbf{b})$. Only a small proportion of the grains release their cytoplasm, and the remaining grains stay intact (b: intact grain on the right). However, in the fragile pollen, PCG release can also occur through breaks of the exine $(\mathbf{c}) . \times 400$.

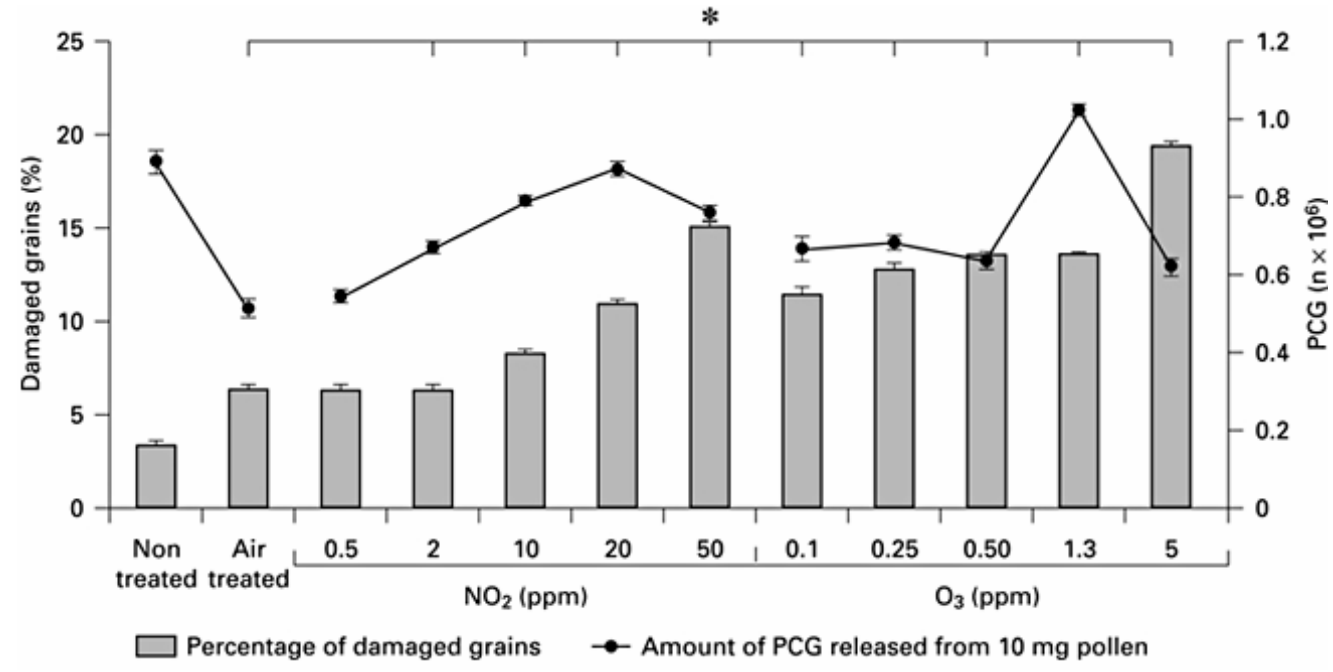

Fig. 3. Effect of exposure to air or pollutants on the proportion of damaged pollen grains and on the release of PCG following contact with water. Pollen samples were exposed to several concentrations of $\mathrm{NO}_{2}$ or $\mathrm{O}_{3}$. The proportion of damaged pollen was observed with ESEM (left axis, bars \pm confidence intervals of probability estimated under the binomial distribution assumption) and the PCG released from the remaining intact grains contained in $10 \mathrm{mg}$ pollen suspended in $500 \mu \mathrm{l}$ of distilled water were counted (right axis, points $\pm \mathrm{SEM}, * \mathrm{p}<0.01, \mathrm{n}=$ $5)$. 


\section{Discussion}

As the number of studies on the effects of air pollution on allergy prevalence increases, it becomes clear that gaseous air pollution affects airways by inducing inflammation, and by playing an adjuvant role in respiratory allergy. On the other hand, it also has an effect on airborne allergens themselves. Pollen allergens release is modified by exposure of pollen to environmental pollution [6]. Here, we showed that air pollution could also induce the release of PCG in our exposure system. Although the damages observed on the pollen in this study come partly from mechanic wear in the exposure process, there was a clear adjuvant effect of the pollutants. In a study from Taylor et al. [15] on Lolium perenne, $72 \%$ of freshly collected pollen grains released their PCG upon contact with water. However, this proportion rapidly decreased to $10 \%$ after pollen was stored for 48 $\mathrm{h}$ at $27^{\circ} \mathrm{C}$, and to $1 \%$ for commercial pollen that has been kept at $4^{\circ} \mathrm{C}$ for more than 1 year. Our experiments were carried out with commercial pollen. Although this does not allow working with pollen having a high capacity to release intrinsic PCG, it exemplifies the homogeneity of the samples and the possibility for other groups to reproduce the experiments. Our results showed that the interaction of pollen with pollutants increases the proportion of pollen grains releasing their PCG upon contact with water. Compared to air-treated samples, an increase of PCG release in pollutant-treated samples was observed in a dose-dependent manner, becoming statistically significant with realistic concentrations: $0.1 \mathrm{ppm} \mathrm{O}_{3}$ is a concentration frequently reached during the summer in polluted areas and large cities (e.g. Paris in July 1994, 0.13 ppm) or industrial zones (e.g. Etang de Berre in the south of France, $0.3 \mathrm{ppm}$ ). Regarding $\mathrm{NO}_{2}, 2 \mathrm{ppm}$ is above the environmental levels but can be reached in indoor environments. Moreover, the first part of the PCG release curve from ozone-treated pollen shows a plateau. This suggests that lower concentrations of ozone might also induce significantly higher PCG release. PCG have been shown to contain allergens [10] and to be able to trigger an IgE-mediated response in rats [11] and asthmatic patients [16]. In previous studies, pollen-specific IgE ELISA inhibition experiments showed that $2.6 \times 10^{6}$ PCG contained as much allergen as in $100 \mu \mathrm{g}$ of pollen. Knowing this, we then observed that for the same amount of allergens, PCG triggered a higher in vitro cellular response than intact pollen or pollen extract [10], indicating that PCG increased the bioavailability of pollen allergens. This may be explained by their particulate nature [17] and/or due to the more efficient presentation by antigen-presenting cells [18]. In addition, the critical role of PCG in grass-pollen-induced respiratory allergy may be due to their size $(3 \mu \mathrm{m})$, which allows them to penetrate the deep airways in contrast to pollen grains (30-60 $\mu \mathrm{m}$ ). Finally, elevated levels of airborne PCG have been reported following light rainfall in the pollen season although intact pollens were no longer detectable, showing the epidemiological relevance of these particles [19]. In this study, we showed that structural damage of pollen grains by traffic-related air pollutants $\left(\mathrm{O}_{3}\right.$ and $\left.\mathrm{NO}_{2}\right)$ could lead to spontaneous release of the PCG. If not broken, the grains became more fragile and the natural expulsion of PCG upon contact with water was enhanced. The mechanisms involved in this phenomenon remain to be elucidated. The pollen wall is composed of sporopollenin, a highly resistant biopolymer. This envelope contains thousands of microcanals linking the inside of the grain to the outside. Thus one can hypothesize that oxidative pollutants affect: (a) the structure of the sporopollenin, making it more fragile, and/or (b) the inside of the pollen, modifying the susceptibility to osmotic shock. Our exposure system remains quite artificial in mimicking the mechanical and chemical stresses encountered by the pollen grains in the atmosphere. However, it provides qualitative information and shows that peaks of traffic-related air pollution during the pollen season can lead to an increased bioavailability of airborne pollen allergens by inducing or facilitating the release of PCG. This mechanism of induction of allergen release might lead to more thunderstorm 
asthma episodes in polluted areas and even provide a new link between air pollution and the increased prevalence of respiratory allergic diseases.

\section{Acknowledgment}

The present study was supported by the French Ministry of the Environment and Sustainable Development (BCRD-DRC-05-AP-2001).

\section{References}

1 Johnston CJ, Reed CK, Avissar NE, Gelein R, Finkelstein JN: Antioxidant and inflammatory response after acute nitrogen dioxide and ozone exposures in C57BI/6 mice. Inhal Toxicol 2000;12:187-203.

2 Galan I, Tobias A, Banegas JR, Aranguez E: Short-term effects of air pollution on daily asthma emergency room admissions. Eur Respir J 2003;22:802-808.

3 Koike $\mathrm{E}$, Watanabe $\mathrm{H}$, Kobayashi T: Exposure to ozone enhances antigen-presenting activity concentration dependently in rats. Toxicology 2004;197:37-46.

4 Barck C, Lundahl J, Hallden G, Bylin G: Brief exposures to $\mathrm{NO}_{2}$ augment the allergic inflammation in asthmatics. Environ Res 2005;97:58-66.

5 Balmes JR: The role of ozone exposure in the epidemiology of asthma. Environ Health Perspect $1999 ; 101: 219-224$.

6 Behrendt H, Becker WM, Fritzsche C, Sliwa-Tomczok W, Tomczok J, Friedrichs KH, Ring J: Air pollution and allergy: experimental studies on modulation of allergen release from pollen by air pollutants. Int Arch Allergy Immunol 1997;113:69-74.

7 Chehregani A, Majde A, Moin M, Gholami M, Ali Shariatzadeh M, Nassiri H: Increasing allergy potency of Zinnia pollen grains in polluted areas. Ecotoxicol Environ Saf 2004;58:267-272.

8 Wilson AF, Novey HS, Berke RA, Surprenant EL: Deposition of inhaled pollen and pollen extract in human airways. N Engl J Med 1973;288:1056-1058.

9 Grote M, Vrtala S, Niederberger V, Wierman R, Valenta R, Reichelt R: Release of allergen-bearing cytoplasm from hydrated pollen: a mechanism common to a variety of grass (Poaceae) species revealed by electron microscopy. J Allergy Clin Immunol 2001;108:109-115.

10 Motta A, Peltre G, Dormans JAMA, Withagen CET, Lacroix G, Bois F, Steerenberg PA: Phleum pratense pollen starch granules induce humoral and cell-mediated immune responses in a rat model of allergy. Clin Exp Allergy 2004;34:310-314.

11 Motta AC, Dormans JA, Peltre G, Lacroix G, Bois FY, Steerenberg PA: Intratracheal instillation of cytoplasmic granules from Phleum pratense pollen induces IgE- and cell-mediated responses in the Brown Norway rat. Int Arch Allergy Immunol 2004;135:24-29.

12 Suphioglu C: Thunderstorm asthma due to grass pollen. Int Arch Allergy Immunol 1998;116:253260.

13 Taylor PE, Jonsson H: Thunderstorm asthma. Curr Allergy Asthma Rep 2004;4:409-413.

14 Marks GB, Colquhoun JR, Girgis ST, Hjelmroos Koski M, Treloar ABA, Hansen P, Downs SH, Car NG: Thunderstorm outflows preceding epidemics of asthma during spring and summer. Thorax $2001 ; 56: 468-471$.

15 Taylor PE, Flagan RC, Valenta R, Glovsky MM: Release of allergens as respirable aerosols: a link between grass pollen and asthma. J Allergy Clin Immunol 2002;109:51-56.

16 Suphioglu C, Singh MB, Taylor P, Bellomo R, Holmes P, Puy R, Knox RB: Mechanism of grasspollen-induced asthma. Lancet 1992;339:569-572.

17 Nygaard UC, Aase A, Lovik M: The allergy adjuvant effect of particles - genetic factors influence antibody and cytokine responses. BMC Immunol 2005;6:11.

18 Currie AJ, Stewart GA, McWilliams AS: Alveolar macrophages bind and phagocytose allergencontaining pollen starch granules via C-type lectin and integrin receptors: implications for airway inflammatory disease. J Immunol 2000;164:3878-3886.

19 Schäppi GF, Suphioglu C, Taylor PE, Knox B: Concentrations of the major birch tree allergen Bet v 1 in pollen and respirable fine particles in the atmosphere. J Allergy Clin Immunol 1997;100:656-661 